\title{
Quasi-Planar Graphs Have a Linear Number of Edges ${ }^{\star}$
}

\author{
Pankaj K. Agarwal ${ }^{1}$, Boris Aronov ${ }^{2}$, János Pach ${ }^{3,4,5}$, Richard Pollack ${ }^{4}$, and \\ Micha Sharir ${ }^{4,6}$
}

1 Department of Computer Science, Duke University, Durham, NC 27708-0129 USA

2 Computer and Information Science Department, Polytechnic University, Brooklyn, NY 11201-3840 USA

3 Department of Computer Science, City College, CUNY, New York, NY, USA

${ }^{4}$ Courant Institute of Mathematical Sciences, New York University, New York, NY 10012, USA

${ }^{5}$ Hungarian Academy of Sciences, Budapest, Hungary

${ }^{6}$ School of Mathematical Sciences, Tel Aviv University, Tel Aviv 69978, Israel

\begin{abstract}
A graph is called quasi-planar if it can be drawn in the plane so that no three of its edges are pairwise crossing. It is shown that the maximum number of edges of a quasi-planar graph with $n$ vertices is $O(n)$.
\end{abstract}

\section{Introduction}

We say that an undirected graph $G(V, E)$ without loops or parallel edges is drawn in the plane if each vertex $v \in V$ is represented by a distinct point and each edge $e \in E$ is represented by a Jordan arc connecting the points corresponding to endpoints of $e$. Throughout this paper, we assume that any two arcs of a drawing have at most one point in common, which is either a common endpoint or a common interior point where the two arcs cross each other. We do not make

\footnotetext{
* Work on this paper by Pankaj K. Agarwal has been supported by NSF Grant CCR93-01259, an NYI award, and matching funds from Xerox Corporation. Work on this paper by Boris Aronov has been supported by NSF Grant CCR-92-11541 and by a Sloan Research Fellowship. Work on this paper by János Pach, Richard Pollack, and Micha Sharir has been supported by NSF Grants CCR-91-22103 and CCR94-24398. Work by János Pach was also supported by Grant OTKA-4269 and by a CUNY Research Award. Work by Richard Pollack was also supported by NSF Grants CCR-94-02640 and DMS-94-00293. Work by Micha Sharir was also supported by NSF Grant CCR-93-11127, by a Max-Planck Research Award, and by grants from the U.S.-Israeli Binational Science Foundation, the Israel Science Fund administered by the Israeli Academy of Sciences, and the G.I.F., the German-Israeli Foundation for Scientific Research and Development. Work on this paper was done during the participation of the first four authors in the Special Semester on Computational and Combinatorial Geometry organized by the Mathematical Research Institute of Tel Aviv University, Spring 1995.
} 
any notational distinction between vertices of $G$ and the corresponding points in the plane, or between edges of $G$ and the corresponding Jordan arcs.

A graph that can be drawn in the plane without crossing edges is planar. We call a graph quasi-planar if it can be drawn in the plane with no three pairwise crossing edges. The aim of this paper is to establish that the number of edges of any quasi-planar graph with $n$ vertices is $O(n)$. This improves an earlier result of Pach et al. [6].

Theorem 1. If $G(V, E)$ is a quasi-planar graph, then $|E|=O(|V|)$.

We prove this theorem in Section 2, and in Section 3 we consider some related problems and generalizations.

\section{Proof of Theorem 1}

To simplify our presentation, we only prove the theorem in the special case when $G$ has a straight-line drawing with no three pairwise crossing edges (straight line segments). Remarkably, the proof for the general case requires only minor modification.

The set of edges $E=E(G)$ defines a cell complex in the plane, whose 0-, 1-, and 2-dimensional cells will be called nodes, segments, and faces, respectively. This cell complex is known as the arrangement of the set of edges of $G$ and is denoted by $\mathcal{A}(E)$. For example, nodes of $\mathcal{A}(E)$ are the endpoints and crossings of graph edges, and a segment of $\mathcal{A}(E)$ is a portion of a graph edge between two consecutive nodes. (To avoid ambiguity we hereafter refer to vertices and edges of $G$, and to the corresponding points and line segments in the plane, as "graph vertices" and "graph edges," respectively.) Let $X$ be the set of crossings of graph edges, $N=V \cup X$ the set of all nodes of $\mathcal{A}(E), S$ the set of its segments, and $F$ the set of its faces. For a face $f \in F$, the complexity $|f|$ of $f$ is the number of segments of $S$ on the boundary $\partial f$ of $f$. As usual, if both sides of an edge are incident to the interior of $f$, then it contributes 2 to $|f|$. Let $t(E)=|\{f \in F:|f|=3\}|$ be the number of triangular faces in $F$.

Lemma 2. Let $G(V, E)$ be a graph drawn in the plane. Then the total complexity of all non-quadrilateral faces of the arrangement $\mathcal{A}(E)$ is at most $4 t(E)+20|V|$.

Proof. It is sufficient to prove the lemma with the assumption that the planar graph $(N, S)$ is connected and $|S|>1$.

Recall the following familiar facts:

$$
\begin{gathered}
\sum_{f \in F}|f|=2|S|, \\
2|S|=\sum_{v \in N} \operatorname{deg}(v)=\sum_{v \in V} \operatorname{deg}(v)+\sum_{v \in X} \operatorname{deg}(v) \geq 2|E|+4|X|,
\end{gathered}
$$

and

$$
|V|+|X|+|F|=|N|+|F|=|S|+2 .
$$


The first two lines just express two different ways of counting the edges of the planar graph $(N, S)$, as the sum of face complexities and of vertex degrees, respectively. The third line is Euler's relation. These easily yield

$$
\sum_{f \in F}|f| \leq 4|V|+4|F|-2|E|-8, \quad \text { hence } \sum_{f \in F}(|f|-4) \leq 4|V| .
$$

Finally, since $|f|>4$ implies $|f| \leq 5(|f|-4)$, we have

$$
\sum_{f \in F,|f| \neq 4}|f|=3 t(E)+5 \sum_{f \in F}(|f|-4) \leq 3 t(E)+20|V| \text {. }
$$

Lemma 3. Let $G(V, E)$ be a quasi-planar graph drawn in the plane. Then the overall complexity of all faces $f$ of $\mathcal{A}(E)$, such that $f$ is either a non-quadrilateral face or a quadrilateral face incident to at least one vertex of $G$, is $O(|V|+|E|)$.

Proof. Note that $t(E)=O(|E|)$, as each triangular face $f$ of $\mathcal{A}(E)$ must be incident to a vertex of $G$. For otherwise there would be three pairwise crossing edges. It is easy to check that the number of faces of $\mathcal{A}(E)$ incident to graph vertices is at most $2|E|$. In addition, this implies that the overall complexity of all quadrilateral faces of $\mathcal{A}(E)$ incident to a graph vertex in $V$ is also $O(|E|)$. The lemma is now an immediate consequence of Lemma 2.

Let $G(V, E)$ be a quasi-planar graph drawn in the plane with $n=|V|$ vertices. Returning to the proof of Theorem 1, we may assume without loss of generality that $G$ is connected, as it suffices to establish a linear bound on the number of graph edges in each connected component of $G$. Let $G_{0}=\left(V, E_{0}\right)$ be a spanning tree of $G$, so $\left|E_{0}\right|=n-1$. Let $E^{*}=E \backslash E_{0}$. Note that each face of the arrangement $\mathcal{A}\left(E_{0}\right)$ is simply connected, for otherwise the union of nodes and segments of $\mathcal{A}\left(E_{0}\right)$ would not be connected, contradicting the connectedness of $G_{0}$. Moreover, by Lemma 3 , the complexity of all faces of $\mathcal{A}\left(E_{0}\right)$, which are either non-quadrilaterals or quadrilaterals incident to a point in $V$, is $O(n)$. We refer to the remaining faces of $\mathcal{A}(E)$ as crossing quadrilaterals.

In the sequel, we use the following notion. A graph is called an overlap graph if its vertices can be represented by intervals on a line such that two vertices are adjacent if and only if the corresponding intervals overlap but neither contains the other [1]. Gyárfás [2] (see also [3]) has shown that every triangle-free overlap graph can be colored by a constant number, $c$, of colors, and Kostochka [4] proved that this is true with $c=5$.

For each graph edge $e \in E^{*}$, let $\Xi(e)$ denote the set of segments of $\mathcal{A}\left(E_{0} \cup\{e\}\right)$ that are contained in $e$. In other words, it is the set of segments into which $e$ is cut by the graph edges from $E_{0}$. By construction, each segment $s \in \Xi(e)$ is fully contained in a face of $f \in \mathcal{A}\left(E_{0}\right)$ and its two endpoints lie on the unique connected component of $\partial f$. For each face $f$ of $\mathcal{A}\left(E_{0}\right)$, let $X(f)$ denote the set of all segments in $\bigcup_{e \in E^{*}} \Xi(e)$ that are contained in $f$, and let $H(f)$ denote the quasi-planar graph whose set of edges is $X(f)$. Since $f$ is simply connected, any 
two segments in $X(f)$ cross each other if and only if their endpoints interleave along the boundary of $f$. By cutting the boundary of $f$ so that it becomes an interval and associating with each segment in $X(f)$ the connected interval along the boundary of $f$ between its endpoints, we obtain a collection of intervals with the property that two elements of $X(f)$ cross if and only if the corresponding intervals overlap and neither is contained in the other. This defines a trianglefree overlap graph on the vertex set $X(f)$. Therefore the segments of $X(f)$ can be colored by at most five colors, so that no two segments with the same color cross each other. (Note that, for a graph edge $e \in E^{*}$, several segments in $\Xi(e)$ may be contained in the same face $f$ and thus belong to the same $X(f)$. These segments may be colored by different colors.)

Let $f$ be a face of $\mathcal{A}\left(E_{0}\right)$ other than a crossing quadrilateral, and let $H_{1}(f)$, $\ldots, H_{5}(f)$ be the monochromatic subgraphs of $H(f)$ obtained by the above coloring. Fix one of these subgraphs, say $H_{1}(f)$, and re-interpret it as a graph whose vertices are the (relative interiors of the) edges of $\partial f$ together with the elements of $V$ on $\partial f$, and whose edges are the segments of $H_{1}(f)$. The resulting graph, $H_{1}^{*}(f)$, is clearly planar. We call a face of $H_{1}^{*}(f)$ a digon if it is bounded by exactly two edges, and we call an edge of $H_{1}^{*}(f)$ shielded if both of the faces incident to it are digons. The remaining edges of $H_{1}^{*}(f)$ are called exposed. Observe that, by Euler's formula, there are at most $O\left(n_{f}\right)$ exposed edges in $H_{1}^{*}(f)$, where $n_{f}$ is the number of vertices of $H_{1}^{*}(f)$, which is at most $2|f|$.

We repeat this analysis for each of the other subgraphs $H_{2}(f), \ldots, H_{5}(f)$, and for all faces $f$ of $\mathcal{A}\left(E_{0}\right)$ other than crossing quadrilaterals. It follows that the number of graph edges $e \in E^{*}$ containing at least one exposed segment (in the graph $H_{i}^{*}(f)$ containing it) is $O\left(\sum_{f}|f|\right)$, where the sum extends over all such faces $f$. By Lemma 3 , this sum is $O(n)$.

It thus remains to bound the number of graph edges in $E^{*}$ with no exposed subsegment; we call these edges shielded, borrowing the terminology used above. If $e$ is a shielded graph edge, then, for each $s \in \Xi(e)$, either $s$ lies in a crossing quadrilateral face of $\mathcal{A}\left(E_{0}\right)$, or else $s$ is shielded in its subgraph. Note that no graph edge $e \in E^{*}$ can consist solely of segments passing through crossing quadrilaterals, as the first and last segments necessarily meet faces of $A\left(E_{0}\right)$ that have at least one graph vertex on their boundary, namely an endpoint of $e$.

\section{Lemma 4. There are no shielded edges.}

Proof. Suppose that $e \in E^{*}$ is shielded. Let $a$ and $b$ be the endpoints of $e$. We claim that there exists a graph edge $e^{+} \in E^{*}$ such that (1) $e^{+}$is a graph edge of $E^{*}$ emanating from $a$ next to $e$, and (2) for each segment $s \in \Xi(e)$, there is a corresponding segment $s^{+} \in \Xi\left(e^{+}\right)$, such that $s$ and $s^{+}$connect the same pair of segments of $\mathcal{A}\left(E_{0}\right)$. Let $s_{1}, \ldots, s_{k}$ denote the segments in $\Xi(e)$, appearing along $e$ in this order.

We prove, by induction on $j$, that the claim holds for $s_{1}, \ldots, s_{j}$. Consider first the case $j=1$. Let $a$ and $b$ be the endpoints of $e$, so that $s_{1}$ is incident to $a$ and $s_{k}$ is incident to $b$. Then $s_{1}$ connects $a$ with some edge $\tau_{1}$ of $\mathcal{A}\left(E_{0}\right)$ (note that for a shielded graph edge $e, s_{1} \neq e$ ). Since $s_{1}$ is shielded, there exists another graph 
edge $e^{+} \in E^{*}$ with a subsegment $s_{1}^{+} \in \Xi\left(e^{+}\right)$that connects $a$ to $\tau_{1}$. Clearly, we can choose $e^{+}$with these properties to be the graph edge emanating from $a$ nearest to $e$, proving the claim for $j=1$.

Suppose next that the assertion is true for $j-1$ and $e^{+}$is the graph edge satisfying the inductive assumption. Suppose that $s_{j}$ connects two segments $\tau_{j-1}$ and $\tau_{j}$ of $\mathcal{A}\left(E_{0}\right)$ such that $u=\tau_{j-1} \cap e$ is the common endpoint of $s_{j-1}$ and $s_{j}$, and $v=\tau_{j} \cap e$ is the other endpoint of $s_{j}$. (If $j=k$ then we take $\tau_{j}$ to be the other endpoint $b$ of $e$.) If $s_{j}$ lies in a crossing quadrilateral face $f$, then, as is easily verified, $e$ and $e^{+}$must cross the same pair of opposite edges of $f$, completing the induction step. Otherwise, since $s_{j}$ is shielded, there is a graph edge $e^{\prime} \in E^{*}$ and a subsegment $s^{\prime} \in \Xi\left(e^{\prime}\right)$ that connects $\tau_{j-1}$ and $\tau_{j}$ on the same side of $s_{j}$ as $e^{+}$. Three cases can arise:

- $e^{\prime}=e^{+}:$The induction step is complete.

- $e^{\prime}$ crosses $\tau_{j-1}$ at a point that lies between $u$ and the crossing with $e^{+}$: Since $G$ is quasi-planar, $e^{\prime}$ cannot cross $e$ or $e^{+}$. Moreover, $e^{l}$ cannot have an endpoint within the interior of the triangle $\Delta$ bounded by $e, e^{+}$, and $\tau_{j-1}$, by the induction hypothesis and the fact that all faces of $\mathcal{A}\left(E_{0}\right)$ are simply connected. Hence, $e^{\prime}$ must end at $a$ and lie inside $\Delta$ near $a$. However, this contradicts the choice of $e^{+}$as the closest neighbor of $e$ near $a$. Thus this case is impossible.

- $e^{+}$crosses $\tau_{j-1}$ at a point that lies between $u$ and the crossing with $e^{\prime}$ : In this case, $e^{+}$cannot cross $s_{j}$ or $s^{\prime}$ or terminate inside $f$. Thus, it must meet $\tau_{j}$. This completes the induction step and hence the proof of the claim.

Note that the same analysis also applies when $j=k$, that is, when $\tau_{j}$ is the endpoint $b$ of $e$. Therefore, $e$ and $e^{+}$have the same pair of endpoints. Contradiction.

As there are no shielded edges, the total number of edges of $E^{*}$, and thus also of $E$, is $O(n)$. This completes the proof of Theorem 1 .

\section{Discussion}

In this section we discuss some consequences of the above results.

Theorem 5. Let $G(V, E)$ be a graph with $n$ vertices that can be drawn in the plane with no four pairwise crossing edges. Then the number of edges of $G$ is $O\left(n \log ^{2} n\right)$.

Proof. We first estimate the number $C$ of crossings between the edges of $G$. Let $e$ be an edge of $G$, and let $G_{e}$ be the subgraph of $G$ consisting of all edges that cross $e$. Then $G_{e}$ is a quasi-planar graph. Thus, by Theorem 1, the number of edges of $G_{e}$ is $O(n)$, which implies that $C=O(n|E|)$. One can then combine this estimate with the analysis in [6], to conclude that $|E|=O\left(n \log ^{2} n\right)$. 
Corollary 6. Let $k \geq 4$ be an integer, and let $G$ be a graph with $n$ vertices that can be drawn in the plane with no $k$ pairwise crossing edges. Then the number of edges of $G$ is $O\left(n \log ^{2 k-6} n\right)$.

Proof. This is an immediate consequence of the analysis in [6], which proceeds by induction on $k$, based on the improved bound of Theorem 5 for $k=4$.

Theorem 5 and Corollary 6 improve the bounds given in [6] by a factor of $\Theta\left(\log ^{2} n\right)$.

There are several interesting problems that are left open in this paper. The first problem is to find the best constant of proportionality in the bound of Theorem 1. A trivial lower bound is roughly $6 n$, obtained by overlaying two edge disjoint triangulations of a point set. The constant 6 can be slightly improved.

Another open problem is as follows. For a quasi-planar graph $G$, let $\chi=\chi(G)$ be the smallest number with the property that the edges of $G$ can be colored with $\chi$ colors, so that the edges in each color class form a planar graph. Clearly, if $G$ has $n$ vertices, then the number of edges of $G$ is at most $3 \chi(G) n$. Thus, a plausible conjecture is that $\chi(G)$ is bounded from above by a constant. Recall that this conjecture is true with $\chi(G) \leq 5$, if there exists a plane drawing of $G$ in which no three edges are pairwise crossing and the vertices are in convex position (see also $[2,3]$ for a weaker constant bound and for related results concerning more general classes of graphs). Moreover, if there exists such a drawing of $G$ in which the vertices lie on two parallel lines, then one can easily show that $\chi(G) \leq 2$. Does there exist a constant upper bound for $\chi(G)$ when all edges of $G$ cross a common line? A weaker conjecture is that there exists a subset $E^{\prime}$ of pairwise noncrossing edges of $G$ such that $\left|E^{\prime}\right| \geq \beta|E|$ for some absolute constant $\beta>0$. The existence of such a subset $E^{\prime}$ would imply, by planarity, that $\left|E^{\prime}\right|=O(n)$, which would provide another proof of Theorem 1 .

\section{Acknowledgments}

We wish to thank Zoltán Füredi, Jiří Matoušek, and Otfried Schwarzkopf for helpful discussions concerning the problem studied in this paper. We also extend our gratitude to Bernd Gärtner and Emo Welzl for bringing this problem to our attention in 1989.

\section{References}

1. M. Golumbic, Algorithmic Graph Theory, Academic Press, New York, 1980.

2. A. Gyárfás, On the chromatic number of multiple interval graphs and overlap graphs, Discrete Math. 55 (1985), 161-166.

3. A. Gyárfás and J. Lehel, Covering and coloring problems for relatives of intervals, Discrete Math. 55 (1985), 167-180.

4. A. V. Kostochka, On upper bounds for the chromatic number of graphs, in: Modeli i metody optimizacii, Tom 10, Akademiya Nauk SSSR, Sibirskoe Otdelenie, 1988, 204-226. 
5. J. Pach and P.K. Agarwal, Combinatorial Geometry, Wiley-Interscience, New York, 1995.

6. J. Pach, F. Sharokhi and M. Szegedy, Applications of the crossing number, Proc. 10th Annual ACM Symp. on Computational Geometry (1994), pp. 198-202. Also to appear in Algorithmica. 